\title{
A Simple State-Based Prognostic Model for Railway Turnout Systems
}

\author{
Omer F. Eker, Fatih Camci, Member, IEEE, Adem Guclu, Halis Yilboga, Mehmet Sevkli, Saim \\ Baskan, Member, IEEE
}

\begin{abstract}
The importance of railway transportation has been increasing in the world. Considering the current and future estimates of high cargo and passenger transportation volume in railways, prevention or reduction of delays due to any failure is becoming ever more crucial. Railway turnout systems are one of the most critical pieces of equipment in railway infrastructure. When incipient failures occur, they mostly progress slowly from the fault free to the failure state. Although studies focusing on the identification of possible failures in railway turnout systems exist in the literature, neither the detection nor forecasting of failure progression has been reported. This paper presents a simple state-based prognostic method that aims to detect and forecast failure progression in electro-mechanical systems. The method is compared with Hidden Markov Model based methods on real data collected from a railway turnout system. Obtaining statistically sufficient failure progression samples is difficult considering that the natural progression of failures in electro-mechanical systems may take years. In addition, validating the classification model is difficult when the degradation is not observable. Data collection and model validation strategies for failure progression are also presented.
\end{abstract}

Index Terms-Fault Diagnosis, Diagnostic expert system, Failure Analysis, Rail transportation maintenance, Forecasting, Prognostics, Remaining useful life estimation, Railway Turnouts, Time Series

\section{NOTATION}

$\phi \quad$ : Constant in exponential degradation model

$\beta$ : Random variable following s-normal distribution

$\mu_{\beta}$ : Mean of $\beta$

$\sigma_{\beta}^{2}:$ Variance of $\beta$

$\lambda$ : HMM Model

$\pi$ : Initial probabilities of HMM

$A_{i, j}^{l}$ : Transition probability from state $i$ to $j$ in level $l$

$B$ : Observation probability distributions

$\mathrm{CH}$ : Calinski-Harabasz cluster validity index

$n_{c}:$ Number of samples clustered in cluster $c$

$z_{c}:$ Center of cluster $c$

$z \quad$ : Center of all clusters

Manuscript received December 25, 2009; revised received March 26, 2010. First published XXXX; current version published XXXX. This research was supported by The Scientific and Technological Research Council of Turkey (TUBITAK) under project number 108M275.

Omer Faruk Eker, Adem Guclu, and Halis Yılboga are students at Fatih University, Istanbul Turkey. (omerfarukeker@hotmail.com, ademguclu@live.com, halis65@gmail.com). Fatih Camci is with Meliksah University, Kayseri Turkey (fcamci@meliksah.edu.tr). Mehmet Sevkli and Saim Baskan are with Fatih University, Istanbul, Turkey. (msevkli@fatih.edu.tr, sbaskan@fatih.edu.tr). Copyright (c) 2009 IEEE. Personal use of this material is permitted. However, permission to use this material for any other purposes must be obtained from the IEEE by sending a request to pubs-permissions @ieee.org. $x_{i} \quad$ : Time series data sample $i$

$k \quad$ : Number of clusters

\section{INTRODUCTION}

It is now an obligation to improve reliability, availability, and safety of railway systems to accommodate increasing passenger and cargo transportation with higher train speeds, greater axle loads, and increased service frequency. According to a report of the European Commission, the passenger and cargo transportation volume in European railways are expected to double and triple respectively in 2020 [1]-[5]. It is obvious that this demand increase cannot be satisfied with only building new railways; the efficiency of existing and new railways should be increased. This could be achieved with minimum cost by increasing the availability of railways, which is directly related with repair and maintenance frequency.

Britain's railway infrastructure operator, Network Rail, was responsible for approximately 14 million minutes of train delay in 2002-2003, costing approximately 560 million GBP [5]. Railway turnout systems are the main component of railway infrastructure that affects the availability of the system [6]. For example in England, 3.4 million GBP is spent every year for the maintenance of turnout systems for $1000 \mathrm{~km}$ of railways [6]. ConditionBased Maintenance (CBM), in which the health of the machine is observed in real time and maintenance decisions are based on the current and forecasted machine health, increases system availability, reliability and safety while reducing operating and support costs [7]-[8]. Thus, the application of CBM on railway turnout systems has critical importance for increasing the efficiency of railways.

Diagnostics and prognostics are the two main components of CBM [7]. Diagnostics, the process of identifying the failure, is basically a classification problem; prognostics, the prediction of the failure time of the component with an incipient failure, is a forecasting problem. Various diagnostic methods have been presented in the literature for many diverse industrial systems [9] [14]. Diagnostic methods on various components of the railway and train have also been reported [15]-[20].

There are three main approaches in the literature for diagnostics on turnout systems: feature-, model- and empirically-based methods. In the first approach, special features that help to identify the failures are defined. For example in [21], three features (i.e., irregularity, location of maximum point, and symmetry) are defined in the time series data. These features are obtained by analyzing the absolute difference between the reference and actual signals. The reference signal is collected from a fault-free system. The absolute difference is in the form of a time series-like reference. Irregularity is defined as an unexpected bump in the absolute difference. The location 
of the maximum point is compared with the maximum point location in the reference data. The symmetry of the absolute difference of the left and right sides of the maximum point location is used as the third feature.

In model-based approaches, a model is defined to characterize the system. Deviation from the model is defined as failure and identified as the difference between the model outcome and actual data. Various methods may be applied. In [22], the unobserved component model, in which an observable variable is written as a function of several unobservable components, is used. In [23], parameters like velocity and air volume are expressed as polynomial functions of pressure in a pneumatic turnout system. Thus, the difference between the actual velocity and/or air volume and their expected values for a given pressure identifies the failure.

In empirically-based approaches, a fault-free sample is considered as a reference signal, and failures are identified based on the resemblance of a given signal to the reference signal. The Kalman filter is used in [24] for noise removal and failure identification based on a reference signal. Ref. [25] presents an expert system for failure identification using resemblance to the reference signal. The resemblance is quantified using simple Euclidian distance and dynamic time warping approaches. A good review for failure detection methods for railway turnouts is given in [26].

Failure diagnosis is an important task; however, it only aims to stop (and schedule a maintenance/repair) the system when a failure is identified or let the system continue to work otherwise. Incipient failures progress slowly [27]. This progression starts from the fault free state and ends at the failure state [28]. The system functions properly and does whatever is expected (albeit maybe with decreasing efficiency) until reaching the failure state. It is obvious that the detection of failure progression is more valuable than its detection after the failure reaches to a point. In addition, it is a prerequisite for prognostics [28], [29].

Studies of prognostics for different systems have appeared in the literature in recent years. Prognostic methods can be separated into three categories: evolutionary, degradation prediction, and state-based prognostics [8]. Evolutionary prognostics involves trending features until a predefined threshold, whereas the degradation calculated with features is predicted with statistical and computational methods in the degradation prediction based prognostics [8]. In the last category, the states representing the failure progression are detected and forecasted.

Hidden Markov Models (HMMs) have been used as state-based prognostics in [28], [30]-[34] with different application areas such as drilling processes, pump systems, and AC motors. The life of a drill-bit is represented by health states, each of which is modeled as a single HMM in [31]-[32]. Health state of a drill-bit is identified with the HMM with highest likelihood value given the observed time series signal. In standard HMM, transition from one state to another is independent of time spent in the current state. This assumption is not true in failure degradation applications and it is relaxed in [30] with semi-Markov concept.

In [28], health states are represented as the top state of Hierarchical HMM, rather than a distinct HMM as in [28], [31], [32]. Monte-Carlo simulation with health state transition probabilities is used in [28] to calculate the remaining useful life (RUL). In [33], frequency and severity of noncatastrophic faults are used to detect RUL of an electric drive. Four methods have been used for fault detection and HMM based framework is presented for prognostics. Second order HMM, in which states depend on the two previous states, is used for prediction of health states in [34]. In [35], concentration of lubrication samples is used to identify the abnormality in diesel engines. Then, a belief network created with expert knowledge is used for prognostics.

This paper presents a simple state-based prognostic (SSBP) method that aims to detect and forecast failure progression in electro-mechanical systems. The method is compared with HMM-based methods using real data collected from a railway turnout system. Failures in electro-mechanical systems mostly occur slowly, and it may take months/years for a failure to occur. Thus, obtaining statistically sufficient samples from the faultfree state to the failure state may require a very long time. In addition, the validation of classification methods requires knowing the real failure progression states. However, observing the real failure progression states is very difficult, if not impossible, in most systems [28]. This paper also presents a solution to these problems. The main contributions of this paper can be summarized as follows:

1. Presenting a simple state-based prognostic method and its comparison with HMM-based prognostics;

2. Application of presented (SSBP) and HHM-based prognostics methods in a railway turnout system;

3. Presenting a data collection strategy for slowly developing failures to model the real failure progression;

4. Proposing a strategy to validate the state estimation method without knowing the real failure progression states.

The organization of the paper is as follows: section II gives the details of a turnout system, section III presents the modeling structure, section IV gives experiments and results with real data collected from a turnout system, and section $\mathrm{V}$ concludes the paper.

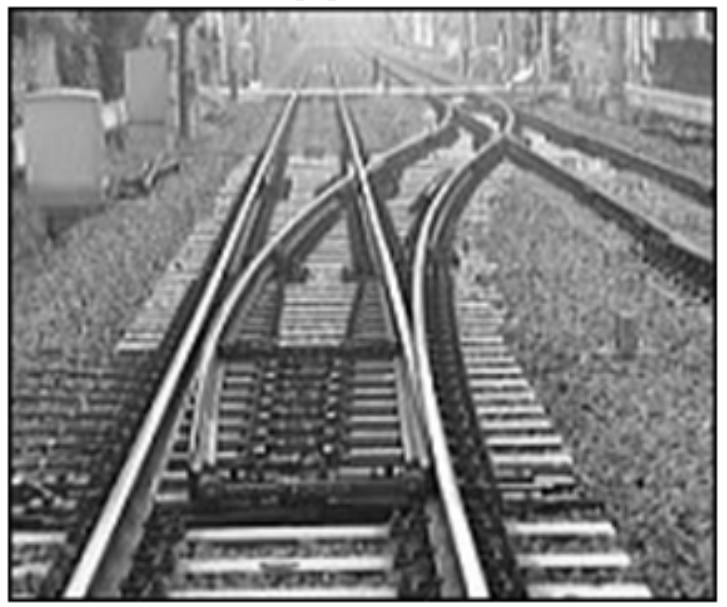

Fig. 1. Railway tracks to be moved by a turnout system 


\section{RAILWAY TURNOUT SYSTEM}

Railway turnout systems allow trains to change their tracks by moving the rails before the train passes, as seen in Fig 1. The turnout system, which is a form of single throw mechanical equipment, is run by a motor as shown in Fig 2. There exist different types of railway turnouts based on motor working principle, such as electromechanic, hydraulic, and pneumatic. The motor moves the blades/switches in two directions, called normal and reverse. This movement takes several seconds. Once the movement is completed, the switches are locked in their position. It is claimed that the turnout systems are the one of the most important component of the railway infrastructure, and there exist examples of accidents caused by turnout failure [6]. This paper focuses on electro-mechanic turnout systems, which consist of a motor, reduction gear, several bearings, drive-detection rods, switches etc.

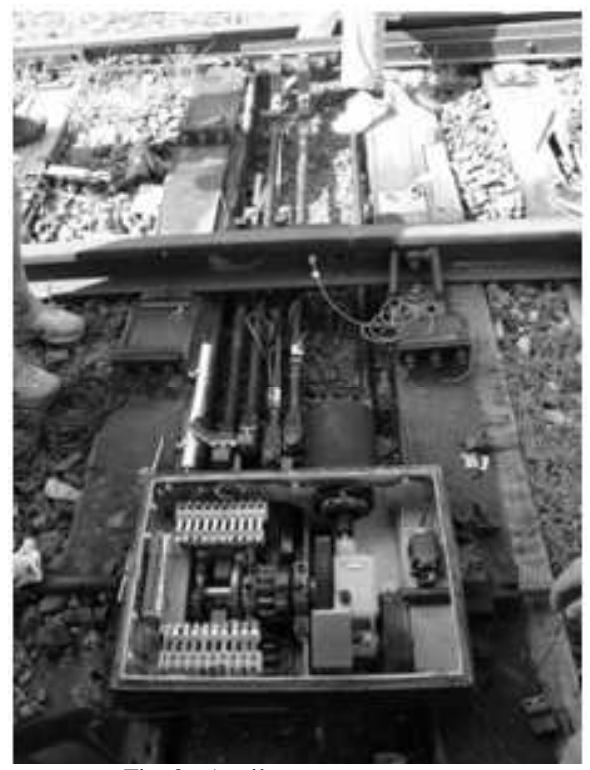

Fig. 2. A railway turnout system

\section{MODELING STRUCTURE}

This section includes two sub-sections: failure progression modeling and a simple state-based prognostic method.

\section{A. Failure Progression Modeling}

Electro-mechanical failures mostly occur slowly, following a degradation path [36]. It is easy to understand the difficulty of obtaining statistically sufficient failure progression samples, considering that a failure may progress for several months to years in real systems. There are two ways to overcome this problem: 1) using prototypes for data collection or 2) creating the failure progression unnaturally.

The prototypes are created with vulnerable materials, and operating conditions are set so that the failure progresses faster than normal [28], [31]-[32]. Choosing thin drill-bits or preventing the proper functioning of cooling systems are some examples used in the literature [28]. Although this approach may be applicable for simple components like a drill-bit, it is difficult to obtain an acceptable prototype to represent something complex like a turnout system. In such a complex system, the failure progression should be created unnaturally.

The exponential degradation model for systems with no prior information about degradation presented in [36] is used in this paper and formulated as in (1). The degradation paths for samples as shown in Fig. 3 are obtained using this formula. Then, the failure time is defined as the point where the degradation reaches a threshold, providing various lifetimes for multiple turnout systems. The degradation level is divided into several groups as illustrated with dashed horizontal lines in the figure. Each group is defined as a state in the failure progression. The time spent in a state corresponds to the length of the $x$-axis (time) of the degradation path (signal) when the $y$-axis falls in that state.

$$
S\left(t_{i}\right)=\phi e^{\left(\beta t_{i}+\varepsilon\left(t_{i}\right)-\frac{\sigma^{2}}{2}\right)}
$$

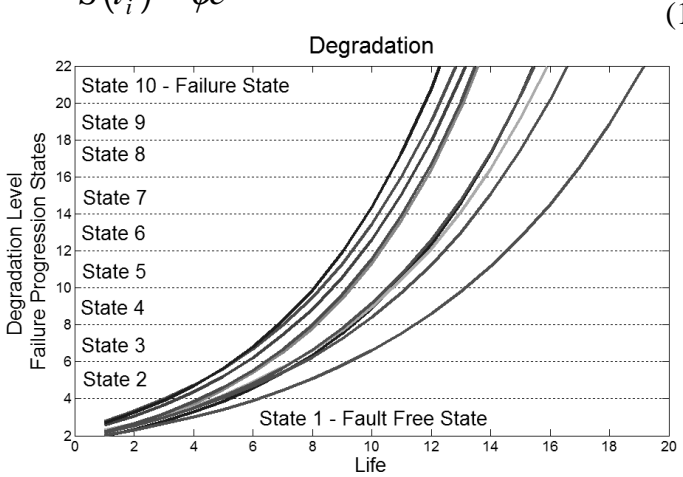

Fig. 3. Failure degradation and discrete health states

After obtaining the life and the time spent in each state for all samples, the data can be collected by creating the failure progression states unnaturally. This could be the size of a crack, how loose a screw has become, the level of wear and tear, or dryness level in the slide chair of the turnout system. When the failure progression is created unnaturally, the data that can be used as representative of that state in different turnout systems, are collected.

\section{B. Simple State-Based Prognostics Method}

The presented method (SSBP) includes three steps, as illustrated in Fig. 4: clustering, cluster evaluation, and expected RUL calculation. For clustering, data from the different health states of multiple systems are clustered using any clustering method. $k$-means clustering is used in this paper due to its simplicity and effectiveness. However, SSBP is independent of the clustering method.

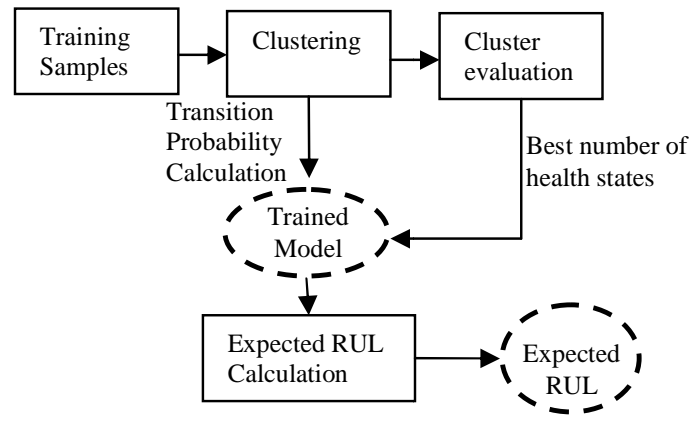

Fig. 4. Prognostics steps

In clustering, the best number of clusters for the given dataset should be identified. In $k$-means clustering, the number of clusters $(k)$ should be given. Although in classification problems parameters can be optimized using 
classification accuracy, clustering problems require different evaluation parameters because the real number of clusters and samples that belong to real clusters are not known. Several cluster validity indexes have been proposed in the literature [37],[38]. Silhouette, DaviesBouldin, Calinski-Harabasz, Dunn, C-index, KrzanowskiLai, and several other indexes are implemented, and Calinski-Harabasz $(\mathrm{CH})$ is chosen as the most robust one for the dataset used in this paper. The formulation of $\mathrm{CH}$ indexes is given in (2).

$$
C H=\left[\frac{\sum_{c=1}^{k} n_{c}\left\|z_{c}-z\right\|^{2}}{k-1}\right] /\left[\frac{\sum_{c=1}^{k} \sum_{i=1}^{n_{c}}\left\|x_{i}-z_{c}\right\|^{2}}{n-k}\right]
$$

The cluster validity index gives the optimal number of clusters and health states in our problem. In a failure progression problem, it is very difficult, if not impossible, to observe the health states the system goes through and to know their total number. Thus, clustering and validity indexes are used to identify the number of health states.

After the current health state of a given system is identified using clustering, the remaining useful life is forecasted as illustrated in Fig. 5. The expected RUL is calculated using the transition probabilities between health states as shown (3). The transition probability is the probability of the current health state to change. The results of the $k$-means clustering are used to identify these probabilities. $k$ number of clusters and the number of transitions from one cluster to another are found with all training samples. For example, assume three turnout systems with health states numbered sequentially from 1 to 4 (from the brand new to the failure state) as shown in Table 1. Each row shows the progression in health states for a turnout system. The three turnout systems fail after 13, 10, and 9 time units. A total of twelve transitions from state 1 (transition to state 2 after 4, 6, and 2 time steps, state 1 otherwise) have happened in three turnouts. Because three state transitions out of twelve from state 1 have happened to state 2 , the transition probability from state 1 to state 2 is initialized as 3/12. Similarly, the state transition probability from state 1 to state 1 (state 3 ) is initialized as $9 / 12(0 / 12)$. Other transition initialization values are calculated similarly.

The current health state of the system is represented by $h_{c}$ in (3) and is obtained using the clustering method. Note that the $\infty$ in the formula is represented as the RUL upper limit in the SSBP. As the upper limit increases, the approximation of the RUL improves. The probability of the RUL of each value between 1 and the RUL upper limit is calculated one by one. For a given RUL, there exist many different combinations of possible health states. For example, if the RUL is given as 5 with 3 health states, the combinations are as follows: 311 (i.e., 3 time unit in state 1 , and 1 time unit in state 2 and state 3 ), 221 , $212,131,122$, and 113 . The number of combinations increases exponentially with the number of health states. To handle the computations, transitions are only allowed from a health state to itself or to a consecutive state. Thus, a state cannot jump to second, third, and so on, consecutive health states directly. The probability of staying in a health state $n$ for $s t_{n}$ times means that the health state transitions to itself $s t_{n}-1$ times and transitions to the consecutive health state one time. The calculation is given in (6).

$$
\begin{gathered}
{\left[R U L \mid s t=h_{c}\right]=\sum_{i=1}^{\infty} i \times P\left(\text { rul }=i \mid s t=h_{c}\right)} \\
P(R U L=i \mid s t=h)=\prod_{n=h}^{\text {fail }} P\left(\text { stay }_{n}=s t_{n}\right) \\
\sum_{n=h}^{\text {fail }} s t_{n}=i \\
P\left(\text { stay }_{n}=s t_{n}\right)=q_{n, n}^{s t_{n}-1}\left(1-q_{n, n}\right)
\end{gathered}
$$

The Monte Carlo simulation with HMM used in [28] does not make the assumption of transition of a state only to itself or the consecutive state. However, SSBP removes some of the complications of HMM. The results of SSBP are compared with those of HMM-based prognostics in section IV. The following subsection discusses the details of Hierarchical Hidden Markov Models (HHMM).

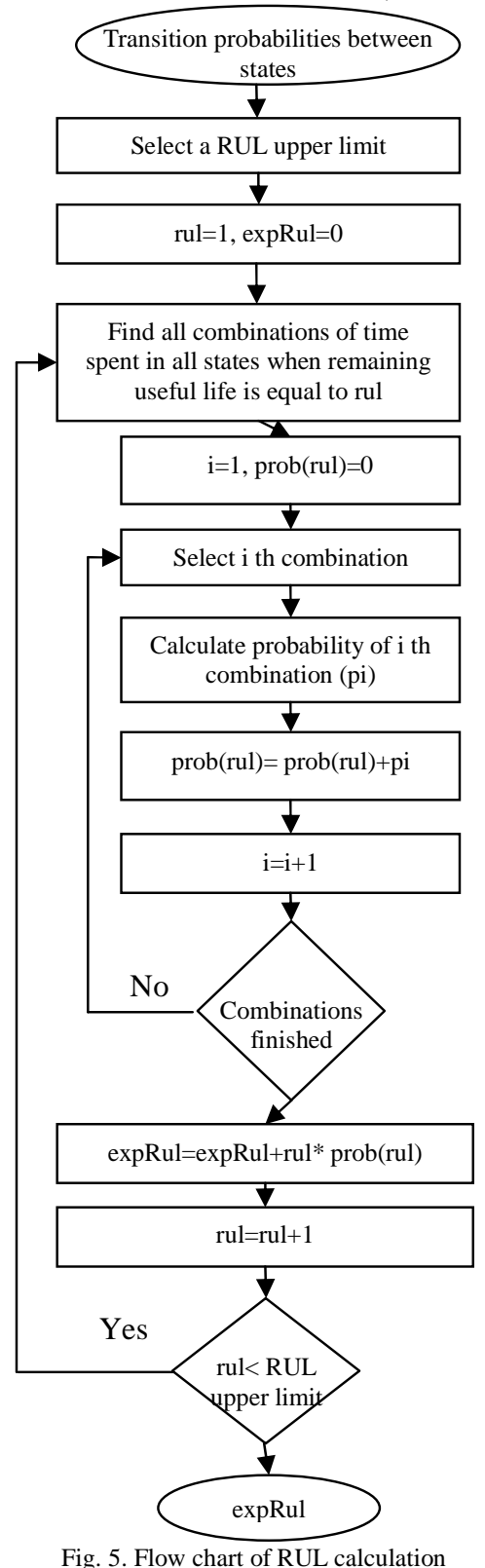

Fig. 5. Flow chart of RUL calculation 


\section{Hierarchical Hidden Markov Model}

The Hierarchical HMM (HHMM) is a version of the Hidden Markov Model (HMM) designed to model hierarchical structures for sequential data. The simplest HHMM includes two hidden layers; the state in the top hidden layer is considered to be a self-contained probabilistic model. In other words, in the simplest HHMM, each state in the top layer is itself an HMM. The observed state is affected by both nodes in the top and low hidden layer in any given time frame. The states in the top hidden layer represent the health states of the turnout system, whereas states in the low hidden layer represent the state of the movement of the rails. In other words, the low level states show the location of the rail during the back and forth movement of the rail. Readers are referred to [8] and [28] for the details of HHMM.

\section{Initialization of HHMM}

An HHMM model is represented as $\lambda(\lambda=(A, B, \pi))$ and includes parameters of initial probabilities $(\pi)$, transition probabilities ( $A$ ), and observation probability distributions $(B)$. The following paragraphs discuss the initialization of parameters in an HHMM.

Initial probabilities $(\pi)$ exist for two hidden variables: top and sub-hidden states. Top hidden states represent the health state of the turnout system. The initial probability of a top state is the probability of a turnout system to be in a given health state for the first usage or after maintenance/repair. This is always 1 for a brand new state (and also after maintenance assuming perfect maintenance) and 0 for other states. The data collected during the movement of the rails with force generated by the turnout system is in the form of a time series and consists of several states (i.e., sub-states). The initial probability of a sub-state is the probability of sub-states in the beginning of the movement. In our experiments, the initial probabilities are assumed to be 1 for the first top and sub-states and 0 for the other states. That is, the perfect maintenance assumption is made, and all turnout movements are assumed to start from the same sub-state.

\begin{tabular}{|l|r|r|r|r|r|r|r|r|r|r|r|r|r|}
\hline & \multicolumn{10}{|c|}{ Life of turnouts } \\
\hline & 1 & 2 & 3 & 4 & 5 & 6 & 7 & 8 & 9 & 10 & 11 & 12 & 13 \\
\hline Turnout1 & 1 & 1 & 1 & 1 & 2 & 2 & 2 & 3 & 3 & 3 & 3 & 4 & 4 \\
\hline Turnout2 & 1 & 1 & 1 & 1 & 1 & 1 & 2 & 3 & 3 & 4 & & & \\
\hline Turnout3 & 1 & 1 & 2 & 2 & 2 & 2 & 3 & 3 & 4 & & & & \\
\hline
\end{tabular}

Table 1. Turnout health states progression examples

The top state transition probabilities are initialized using the transition probabilities obtained using $k$-means clustering as discussed above. The other transition probability parameter is the probability of the current substate to change. To initialize this parameter, the time series data collected during the rail movement are divided into equal number of successive groups: $s$ that also is the number of sub-states. Then $A_{i, j}^{2}$ is initialized as follows: $A_{i, i+1}^{2}=1 / s, \quad A_{i, i}^{2}=1-1 / s$, and $A_{i, j}^{2}=0 \quad \forall i, j$ $i \neq j$ and $j \neq i+1$.

The last parameter to be initialized is the observation probability distribution parameter, $B_{i, j}$, which represents the distribution parameters of the observed parameter under health state $i$ and sub-state $j$. To initialize $B_{i, j}$, the data points that are collected from a turnout system with each health state and sub-state are categorized. The data from all of the $h \times s$ categories are obtained, where $h$ and $s$ are the number of health and sub-states, respectively. Then, the distribution parameters (i.e., mean and standard deviation) of the data in each category are calculated and used as initialization values for $B_{i, j}$.

\section{EXPERIMENT \& RESULTS}

The presented method (SSBP) is applied to data collected from a turnout system of the Turkish State Railways located in Babaeski, Tekirdag. The following subsections discuss the data collection, failure progression, and results of the SSBP.

\section{A. Data Collection}

The system used for data collection is an electromechanical type turnout with two drive rods, one for each rail. Nine sensors were installed in the turnout system: two force sensors for each drive rod, one current, two voltage (separate sensors for forward and backward movement), two proximity sensors for each rail and two linear rulers for each drive rod. The most commonly used sensors in the literature are current and force sensors [24]. Force sensors are electrical sensing devices that are used to measure tension and compression forces. Tension cells are used for measuring a straight-line force "pulling apart" along a single axis; the force is typically denoted as positive. Compression tension cells are used for measurement of a straight-line force "pushing together" along a single axis; the force is typically annotated as negative. Current sensors measure DC current levels. They receive current inputs and provide outputs as analog voltage signals, analog current levels, switches, or audible signals. Voltage sensors are used to measure voltage in electric circuits. Proximity sensors measure the distance between the stock rail and switch rail of railway turnout systems. A linear position measuring sensor is installed on stretchers of the turnout system and measures the linear position of the switch rails. Time series data are acquired from both normal to reverse and reverse to normal movements of a turnout system. Figs. 6 shows the sensors installed in turnout system.

\section{B. Failure Progression Mechanism}

There are multiple failure modes in a turnout system. The dry slide chair failure mode is the most frequently occurring failure in turnout systems [22]. Slide chairs are the metal platforms installed on twelve wooden traverses, on which the rail is located, as illustrated in Fig. 7. Slide chairs are oiled periodically and in a fault-free system these slide chairs are oily.

Switch rails move back and forth on the slide chairs on the twelve traverses. They move around $17.5 \mathrm{~cm}$ on the slide chair located on the traverse closest to the turnout system. As the traverse gets far away from the turnout system, the movement on that traverse gets smaller. When dry/contaminated slide chair failure modes occur, the friction in the slide chair increases, leading to increased force in the force sensors and changes in voltage and current signals in the motor. In addition, the time to complete the movement may increase. 
The failure progression from the fault-free health state to the failure health state is modeled using the exponential degradation mentioned. The natural progression of the failure mode occurs over a long period of time due to weather and environmental effects. Thus, it is not practical to wait and observe the natural progression of the failure mode. In addition, if the failure progresses naturally, the observation of health states becomes very difficult, if not impossible. In this work, the progression of the dry slide chair failure mode is modeled.
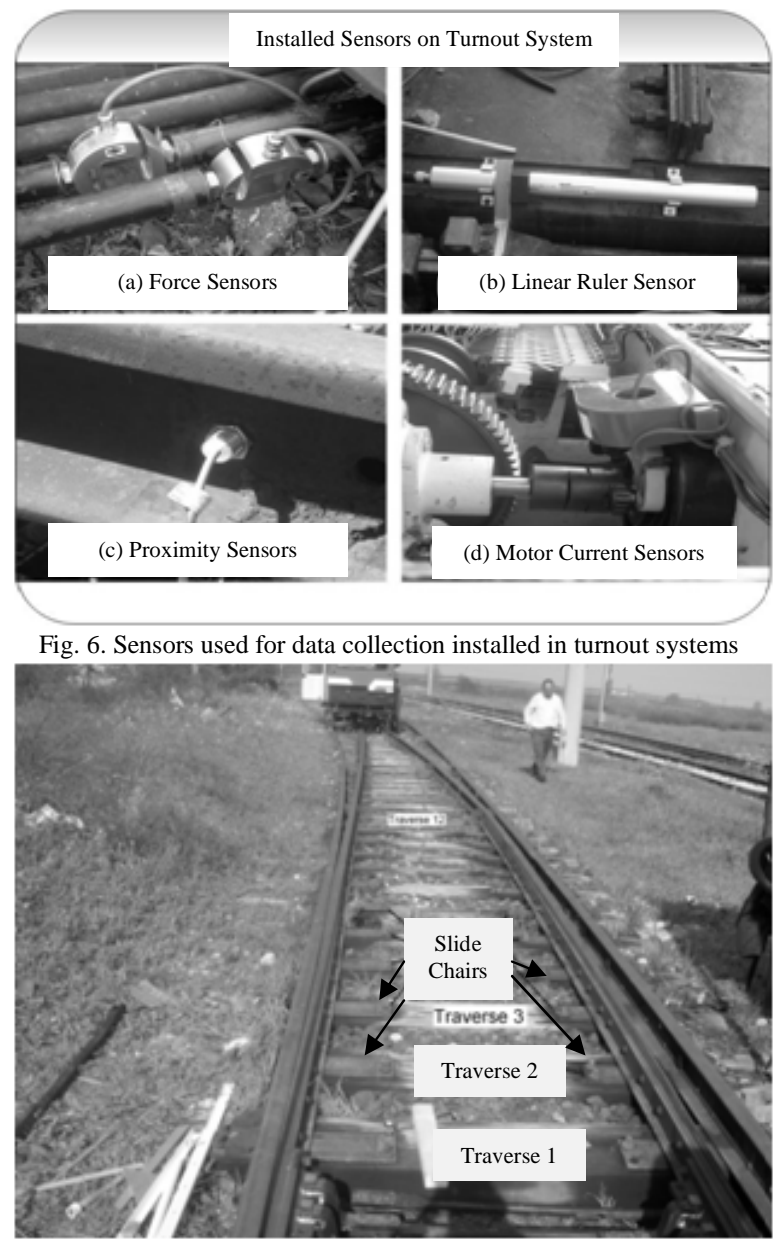

Fig. 7. Modeling the Dry/Contaminated Slide Chair Failure Mode

The failure state is defined as the state when all twelve slide chairs are dry or contaminated. The slide chairs have not been lubricated for a long time to obtain the failure state. The data from the failure state are collected. Then, the slide chairs in the three farthest traverses from the turnout system are oiled, and data are collected. Then, the next farthest traverse is oiled, and data are collected. The oiling and collection of data is repeated one by one from the farthest to the closest slide chairs of the turnout system until all slide chairs are oiled. All of the slide chairs are oily in the final state, which represents the fault-free state. This is achieved in ten steps, each step representing a health state in the failure progression. As a result, many samples from ten health states are obtained. At this point, the life of a turnout system and the time spent in each health state in the failure progression are obtained using exponential degradation modeling with the following parameters: $\phi=1, \mu_{\beta}=0.8 \quad \sigma_{\beta}=0.1$.

An example of current and force signals among ten turnout systems that progress from fault-free to failure states with various lives is shown in Fig 8. As seen from this figure, 13 samples are collected from this turnout system from fault-free to failure state. In other words, its life is 13 units. One can observe the change in the current and force signals as the failure progresses.

This paper uses only the dry slide chair failure mode for the case study and all other conditions, such as operating conditions and wear in the components of the turnout system, are assumed to remain constant. This is a reasonable assumption because all the data are collected in one day, and the dry slide chair failure mode is obtained unnaturally as mentioned above.
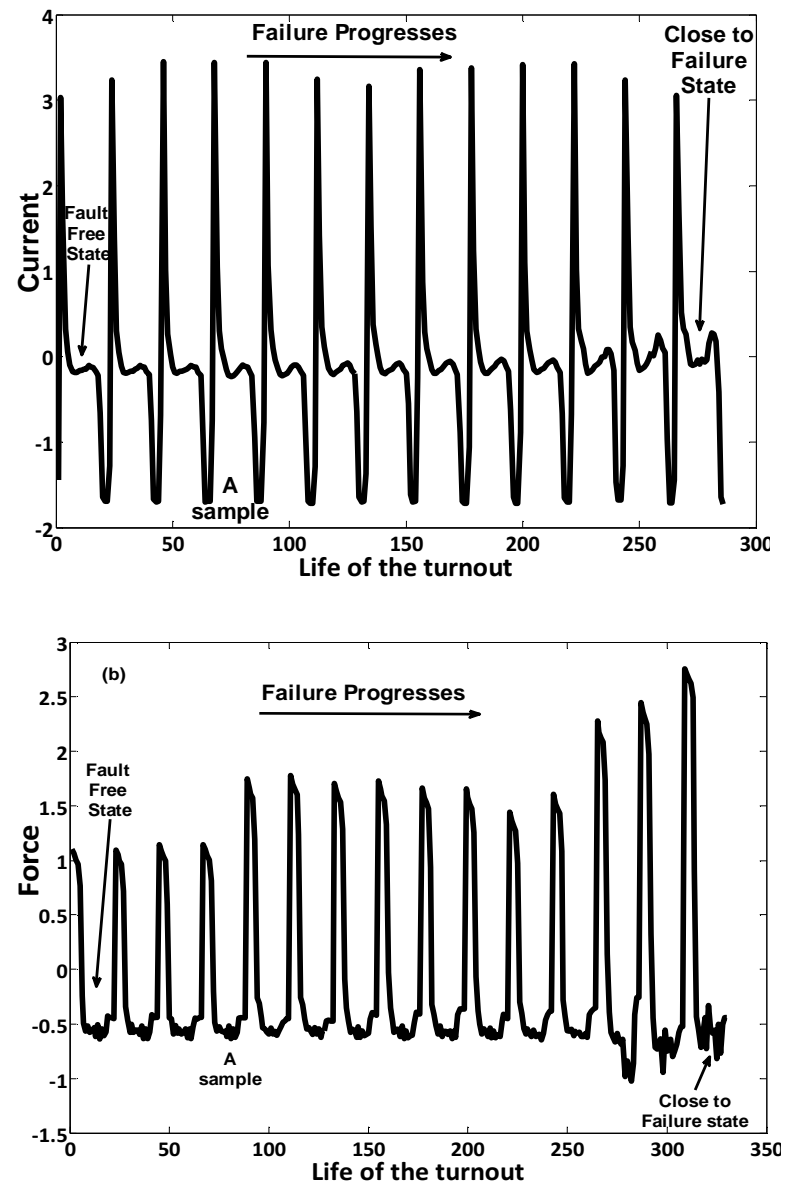

Fig. 8. a) Current b) Force signals with failure progression from 'fault free' state to 'close to failure'state

\section{Results}

The samples are divided into training and testing groups, with $80 \%$ of the samples in the training and the rest in the testing group. The training data are clustered using the number of clusters from two to ten, and the $\mathrm{CH}$ cluster validity index is calculated and displayed in Fig. 9. The maximum of the evaluation function (which indicates the best number of clusters found) occurs at eight clusters. Thus, failure progression should be modeled with eight health states. Note that when the failure progression was modeled, ten unnatural health states were used. When analyzing the health state clustering results, it is observed that health states 8,9 , and 10 are combined into one health state. This is logical considering the variance increase in the states close to failure. However, the best health state number found will be checked with 
forecasting effectiveness to validate the result obtained with the cluster validity index.

Two models (i.e., the SSBP and the HHMM model) are analyzed: samples from the training and testing data are selected, the health states are identified and health state forecasting is performed for both methods. Transition probabilities from both models are extracted, and the expected remaining useful life is calculated as discussed in section III.

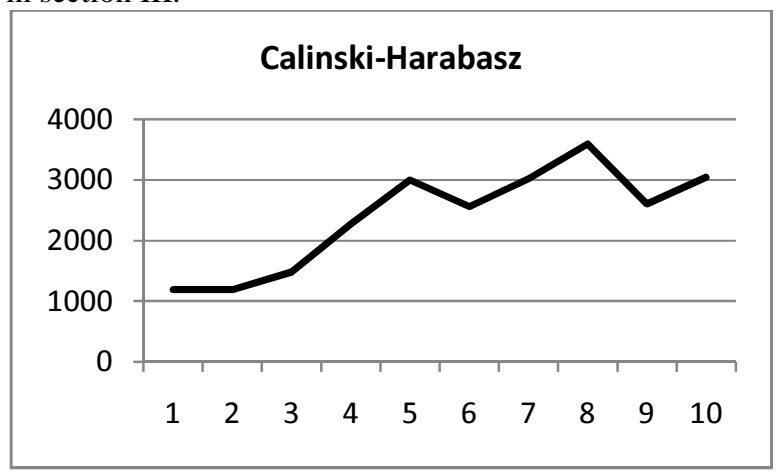

Fig. 9. Evaluation of the number of clusters

The effectiveness of the estimated RUL $\left(\mathrm{RUL}_{\mathrm{e}}\right)$ is calculated by comparing the $\mathrm{RUL}_{\mathrm{e}}$ with the real RUL $\left(\mathrm{RUL}_{\mathrm{r}}\right)$. The real and estimated RULs calculated using HHMM-based prognostics and the SSBP are displayed for ten turnout systems in Fig. 10. Each graph represents one of the turnout systems. The $x$-axis represents the age of the system, whereas the $y$-axis represents the remaining useful life of the system. The dashed line represents the real RUL of the system given the current age. Note that the sum of the $x$-axis and $y$-axis for the real RUL gives the life of the system. The solid line represents the estimated RUL.

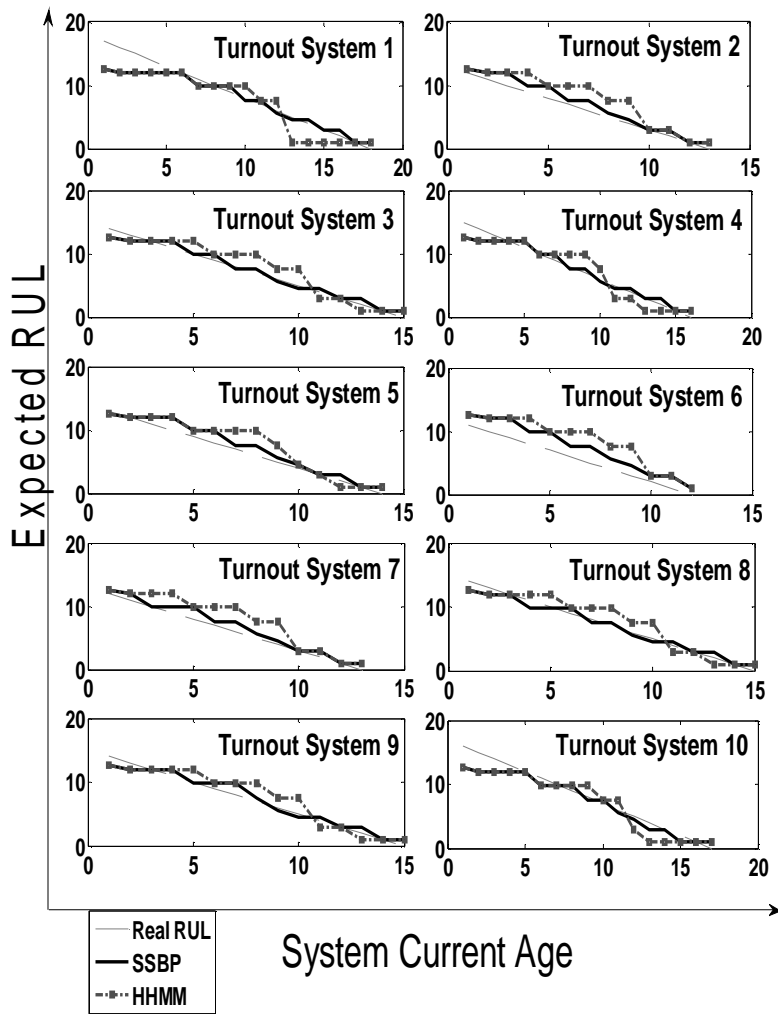

Fig. 10. $\mathrm{RUL}_{\mathrm{r}}$ and $\mathrm{RUL}_{\mathrm{e}}$ for ten turnout systems with HHMM and SSBP
The root mean square error (RMSE) and r-square values between real RUL (RUL $)$ and $\mathrm{RUL}_{\mathrm{e}}$ are used as measures to evaluate the effectiveness of the prognostic method in [26] and [30]. Figs. 11 displays the RMSE (Fig 11.a) and r-square (Fig 11.b) between the RUL $\mathrm{R}_{\mathrm{r}}$ and $\mathrm{RUL}_{\mathrm{e}}$ obtained with HHMM and SSBP. The $x$-axis represents the turnout systems, whereas the $y$-axis gives the RMSE and r-square values. It was expected that HHMMs would outperform the presented simple prognostics method, but the opposite was true. The RMSE values are smaller and the r-square values are greater for the presented method. One can conclude that the optimization within HHMM during training makes the parameters worse from a prognostics perspective, considering its design and technical requirements.

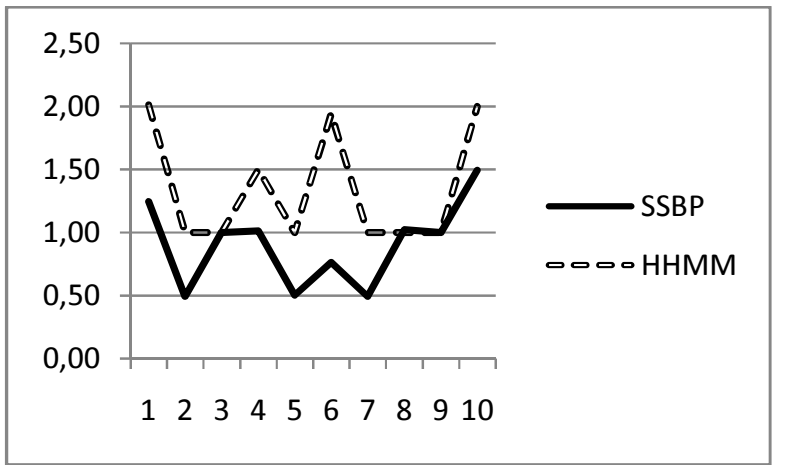

(a)

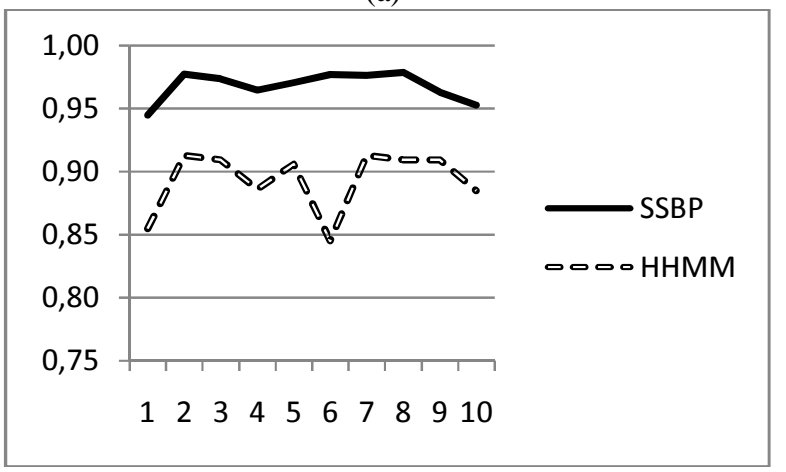

(b)

Fig. 11. Comparison of SSBP and HHMM using a) RMSE b) Rsquare

The presented method is very simple and outperforms HHMM-based prognostics. As a disadvantage, the presented method has restrictions on state transitions that allow transition of a state only to itself and a consecutive state.

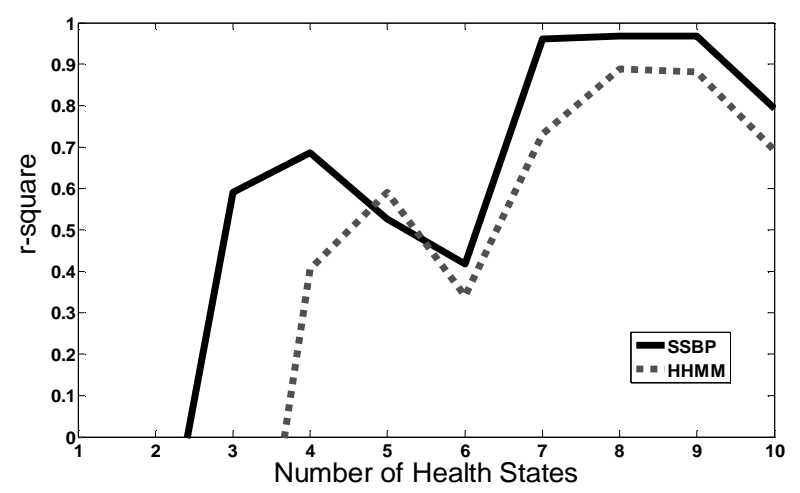

Fig 12. r-square values for different numbers of health states 
Fig. 12 displays the r-square values of $\mathrm{RUL}_{\mathrm{r}}$ and $\mathrm{RUL}_{\mathrm{e}}$ for different health states. The r-square values are negative for the second and third health states and starts increasing thereafter. The highest value obtained is with the eighth and ninth health states, and the r-square value starts decreasing beyond the tenth health state. Note that eight health states were recommended by the $\mathrm{CH}$ cluster validity index. Thus, the prognostics results support the result of the $\mathrm{CH}$ cluster validity index.

\section{CONCLUSION}

Failures often go through several states before making the system unusable. It is critical to identify and forecast these health states as the failure progresses for effective usage of the system. Railway turnouts are one of the most important components of railway infrastructure. Although several studies on failure identification in turnout systems are present in the literature, health state estimation and forecasting have not been reported. One of the most important difficulties in failure progression analysis is the inability to observe the natural progression of failures due to time constraint. Failures occur slowly and obtaining statistically enough failure progression data may take years. This paper presents a strategy to collect data with a given failure degradation model. Then, a simple prognostic method (SSBP) is presented. SSBP and HHMM based prognostic methods are applied to data obtained from a turnout system in the Turkish State Railway and their results are compared. The presented method outperforms the HHMM-based prognostics in the paper. In addition, a validation strategy for health state estimation is presented in the paper.

\section{REFERENCES}

[1] EU Corporate Responsibility Report, 2005

[2] Directive 2001/12/EC of the European Parliament and of the Council of 26 February 2001 amending Council Directive 91/440/EEC on the development of the Community's railways Official, L75 of 15 March 2001, SIN 1012-9200

[3] Directive 2001/13/EC of the European Parliament and of the Council of 26 February 2001 amending Council Directive 95/18/EC on the licensing of railway undertaking, L75 of 15 March 2001, SIN 1012-9200

[4] Directive 2001/14/EC of the European Parliament and of the Council of 26 February 2001 amending Council Directive $91 / 440 /$ EEC on the allocation of railway infrastructure capacity and the levying of charges for the use of railway infrastructure and safety certification, L75 of 15 March 2001, SIN 1012-9200

[5] Garcia Marquez, F., Lewis, W.R., Tobias, M.A., Roberts, C., Life cycle costs for railway condition monitoring, Transport Res. Part E., vol. 44 no. $6,1175-1187,2008$

[6] Fausto Pedro Garcia Marquez, Paul Weston, Clive Roberts, Failure analysis and diagnostics for railway trackside equipment, Engineering Failure Analysis 14, 1411-1426, 2007

[7] A.K.S. Jardine, D. Lin, and D. Banjevic, "A review on machinery diagnostics and prognostics implementing condition-based maintenance", Mechanical Systems and Signal Processing, vol.20, no. 7, 1483-1510, 2006

[8] F. Camci, R. B. Chinnam, Process Monitoring, Diagnostics and Prognostics in Machining Processes, LAP Lambert Academic Publishing, 978-3838335667, Jan. 2010

[9] Y. Wenxian, P. J. Tavner, C.J. Crabtree, M. Wilkinson, "CostEffective Condition Monitoring for Wind Turbines", IEEE Transactions on Industrial Electronics, vol. 57 no. 1, 263-271, 2010

[10] A. Khezzar, M. El Kamel Oumaamar, M. Hadjami, M. Boucherma, H. Razik, "Induction Motor Diagnosis Using Line Neutral Voltage Signatures", IEEE Transactions on Industrial Electronics, vol. 56 no. 11, 4581 - 4591, 2010
[11] F. Immovilli, M. Cocconcelli, A. Bellini, R Rubini "Detection of Generalized-Roughness Bearing Fault by Spectral-Kurtosis Energy of Vibration or Current Signals", IEEE Transactions on Industrial Electronics, vol. 56 no.11, 4710 - 4717, 2009

[12] Z. Pinjia, D. Yi, D. Jing, T. G. Habetler, L. Bin "ImpairedCooling-Condition Detection Using DC-Signal Injection for SoftStarter-Connected Induction Motors", IEEE Transactions on Industrial Electronics, vol. 56 , no. 11, 4642 - 4650, 2009

[13] S. H. Kia, H. Henao, G. A. Capolino, "Torsional Vibration Effects on Induction Machine Current and Torque Signatures in GearboxBased Electromechanical System" IEEE Transactions on Industrial Electronics, vol. 56, no.11, 4689 - 4699, 2009

[14] M. Pineda-Sanchez, M. Riera-Guasp, J.A. Antonino-Daviu, J. Roger-Folch, J. Perez-Cruz, R. Puche-Panadero, "Instantaneous Frequency of the Left Sideband Harmonic During the Start-Up Transient: A New Method for Diagnosis of Broken Bars", IEEE Transactions on Industrial Electronics, vol. 56 no.11 4557 - 4570, 2009

[15] Guzinski, J.; Diguet, M.; Krzeminski, Z.; Lewicki, A.; Abu-Rub, H.; Application of Speed and Load Torque Observers in HighSpeed Train Drive for Diagnostic Purposes, IEEE Transactions on Industrial Electronics, 56(1), 2009, 248 - 256

[16] Stefani, A.; Bellini, A.; Filippetti, F.; Diagnosis of Induction Machines' Rotor Faults in Time-Varying Conditions IEEE Transactions on Industrial Electronics, 56(11), 2009, 4548 - 4556

[17] Cruz, S.M.A.; Stefani, A.; Filippetti, F.; Cardoso, A.J.M.; A New Model-Based Technique for the Diagnosis of Rotor Faults in RFOC Induction Motor Drives, IEEE Transactions on Industrial Electronics, 55(12), 2008, 4218 - 4228

[18] S. Hedayati Kia, H. Henao, G.A. Capolino, "Mechanical Transmission and Torsional Vibration Effects on Induction Machine Stator Current and Torque in Railway Traction Systems," Conference Record of the 1st ECCE, San Jose (USA), 21-24 September 2009, vol. CD-ROM, pp. 3182-3187.

[19] S. Hedayati Kia, H. Henao, G.A. Capolino, "Torsional Vibration Assessment in Railway Traction System Mechanical Transmission," Proceedings International Symposium on Diagnostics for Electrical Machines, Power Electronics \& Drives (SDEMPED'2009), Cargese (France), 31 August-3 September 2009, vol. CD-ROM, 7 pages.

[20] S. Hedayati Kia, H. Henao, G.A. Capolino, "Mechanical Health Assessment of a Railway Traction System," Proceedings IEEEMELECON'08, Ajaccio (France), 5-7 May 2008, pp.453-458.

[21] Marquez F. P. G., Schmid F., Collado J. C., A Reliability centered approach to remote condition monitoring: A railway points case study, Reliability Engineering and System Safety, 80, (2003), 3340

[22] Marquez F. P. G., D. J. P. Tercero, Schmid F., Unobserved component models applied to the assessment of wear in railway points: A case study., European Journal of Operation Research, 176 (2007), 1703-1712

[23] C. Roberts, H.P.B. Dassanayake, N. Lehrasab, C.J. Goodman: Distributed quantitative and qualitative fault diagnosis: Railway junction case study, Control Engineering Practice, 10(4), 2002, 419-429

[24] Marquez F. P. G., Schmid F., A Digital Filter Based Approach to the Remote Condition Monitoring of Railway Turnouts, Reliability Engineering and System Safety, 92, (2007), 830-840

[25] V. Atamuradov, F. Camci, S. Baskan, M. Sevkli, "Failure Diagnostics for Railway Point Machines Using Expert Systems ", The 7th IEEE International Symposium on Diagnostics for Electric Machines, Power Electronics \& Drive, Cargese, France, 2009

[26] Marquez F. P. G., Roberts C., Tobias A. M, Railway point Mechanisms: condition monitoring and fault detection, Proc. IMechE Vol. 223 Part F: Journal of Rail and Rapid Transit, doi: 10.1243/09544097JRRT289

[27] C. Kwan, X. Zhang, R. Xu, L. Haynes, A novel approach to fault diagnostics and prognostics, Proceedings of ICRA '03: IEEE International Conference on Robotics and Automation. 1(3), September 2003, 604-609

[28] Camci F., Chinnam R. B., "Health-State Estimation and Prognostics in Machining Processes", IEEE Transactions on Automation Science and Engineering, doi: 10.1109/TASE.2009.2038170

[29] Yali Xiong; Xu Cheng; Shen, Z.J.; Chunting Mi; Hongjie Wu; Garg, V.K.; Prognostic and Warning System for Power-Electronic Modules in Electric, Hybrid Electric, and Fuel-Cell Vehicles, 
IEEE Transactions on Industrial Electronics, 55(6), June 2008, $2268-2276$

[30] M. Dong, D. He, A segmental hidden semi-Markov model (HSMM) -based diagnostics and prognostics framework and methodology, Mechanical Systems and Signal Processing, 21, 2007, 2248-2266

[31] P. Baruah, P and R.B. Chinnam, R.B., HMMs for diagnostics and prognostics in machining processes, International Journal of Production Research, 43(6), 2005, 1275-1293

[32] R. B. Chinnam, P. Baruah, Autonomous diagnostics and prognostics in machining processes through competitive learningdriven HMM-based clustering, International Journal of Production Research, 47 (23), 2009, 6739 - 6758

[33] E.G. Strangas; S. Aviyente; S. S. H. Zaidi, "Time-Frequency Analysis for Efficient Fault Diagnosis and Failure Prognosis for Interior Permanent-Magnet AC Motors", IEEE Transactions on Industrial Electronics, vol. 55 no. 12, 4191 - 4199, 2008

[34] Morgan, I.; Honghai Liu; Predicting Future States With $n$ Dimensional Markov Chains for Fault Diagnosis, IEEE Transactions on Industrial Electronics, vol. 56 no. 5, 1774 - 1781, 2009

[35] I. Morgan; H. Liu; B. Tormos; A. Sala, "Detection and Diagnosis of Incipient Faults in Heavy Duty Diesel Engines", IEEE Transactions on Industrial Electronics, doi:10.1109/TIE.2009.2038337

[36] N. Gebraeel, A. Elwany J. Pan, "Residual Life Predictions in the Absence of Prior Degradation Knowledge", IEEE Transactions on Reliability, 58(1), 2009, 106-116

[37] U. Maulik, S. Bandyopadhyay, "Performance Evaluation of Some Clustering Algorithms and Validity Indices" IEEE Transactions on Pattern Analysis and Machine Intelligence, 24(12), 2002, 16501654

[38] R.J.B. Campello, E.R. Hruschka, "A fuzzy extension of the silhouette width criterion for cluster analysis", Fuzzy Sets and Systems, 157(21), 2858-2875, 2006

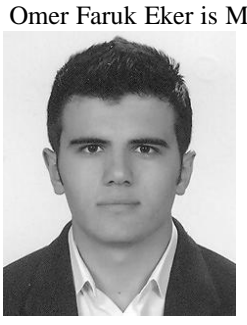

Sc. student in Computer Engineering Departmen at Fatih University and works as a research assistant in the project (108M275) funded by TUBITAK. He received his B.Sc. degree in Department of Secondary School Science and Mathematics Education from Marmara University in Istanbul, Turkey. His research interests include failure diagnostics and prognostics of electro-mechanical systems by using Classification/Clustering and Time Series Analysis techniques.

Fatih Camci received the B.S. degree in computer engineering from

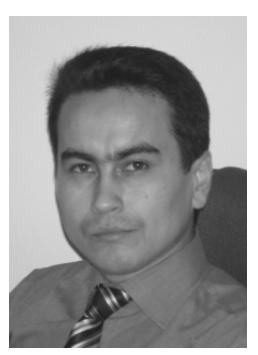
Istanbul University, Istanbul, Turkey, the M.S. degree in computer engineering from Fatih University, Istanbul, and the Ph.D. degree in industrial engineering from Wayne State University, Detroit, MI. $\mathrm{He}$ is an Assistant Professor in computer engineering department at Meliksah University. He worked in several projects related to prognostic health management in USA and Turkey. His expertise includes prognostics health management, intelligence engineering systems, engineering optimization, and video processing.
Adem Guclu is B.Sc. student in Computer Engineering Department at

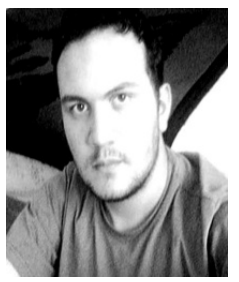
Fatih University. His research interests include Artificial Intelligence, Machine Learning, Pattern Recognition, Data Mining and Web Application Architectures and Development.

Halis Yilboga was born in Van, Turkey in 1986. He is a B.Sc. student in

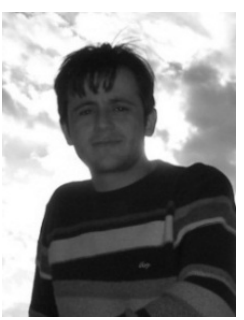
Computer Engineering Department at Fatih University. His research interests include Artificial Intelligence, Machine Learning, Natural Language Processing, Pattern Recognition, and Distributed Systems.

Mehmet Sevkli is an Assistant Professor in Industrial Engineering

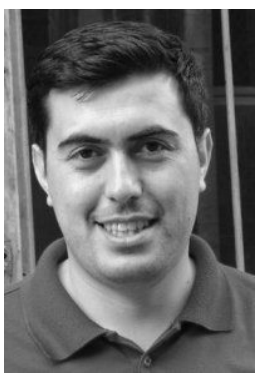
Department, Fatih University, Istanbul, Turkey $\mathrm{He}$ received his B.S. in Industrial Engineering from Sakarya University, his M.Sc. in Industrial Engineering from Fatih University and his Ph.D. in Industrial Engineering, from Istanbul Technical University. His research interests include Meta-heuristics algorithms applied to the combinatorial optimization problem and Multi Criteria Decision Making Methods.

Saim Baskan is an Assistant Professor in Electrical Engineering Department at Fatih University. He received his Ph.D. in Engineering

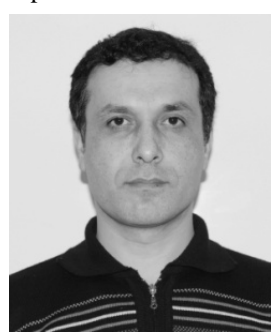
Science from Rensselaer Polytechnic Institute in New York, USA. He received his M.S degree in Electric Power Engineering from Rensselaer Polytechnic Institute. He received his B.S. degree Electrical Education from Gazi University in Turkey. He has worked as Senior Project Engineer at Omega Advanced Solutions in New York, USA. He has been involved in mechanical testing system design. His expertise includes motor control, data acquisition, and sensor less motor control. 